\title{
Functional network motifs defined through integration of protein-protein and genetic interactions
}

\author{
Amruta Sahoo ${ }^{1}$, Sebastian Pechmann ${ }^{\text {Corresp. } 2}$ \\ ${ }^{1}$ Departement de Biochimie, Universite de Montreal, Montreal, QC, Canada \\ ${ }^{2}$ Sebastian Pechmann Research Lab, Saarbruecken, Germany \\ Corresponding Author: Sebastian Pechmann \\ Email address: sebastian@pechmannlab.net
}

Cells are enticingly complex systems. The identification of feedback regulation is critically important for understanding this complexity. Network motifs defined as small graphlets that occur more frequently than expected by chance have revolutionized our understanding of feed- back circuits in cellular networks. However, with their definition solely based on statistical over-representation, network motifs often lack biological context, which limits their usefulness. Here, we define functional network motifs (FNMs) through the systematic integration of genetic interaction data that directly informs on functional relationships between genes and encoded proteins. Occurring two orders of magnitude less frequently than conventional network motifs, we found FNMs significantly enriched in genes known to be functionally related. Moreover, our comprehensive analyses of FNMs in yeast showed that they are powerful at capturing both known and putative novel regulatory interactions, thus suggesting a promising strategy towards the systematic identification of feedback regulation in biological networks. Many FNMs appeared as excellent candidates for the prioritization of follow-up biochemical characterization, which is a recurring bottleneck in the targeting of complex diseases. More generally, our work highlights a fruitful avenue for integrating and harnessing genomic network data. 


\section{Functional network motifs defined through integration of 2 protein-protein and genetic interactions}

3

4

5

6 Amruta Sahoo $^{1}$, Sebastian Pechmann ${ }^{2}$

7

8

$9{ }^{1}$ Département de Biochimie, Université de Montréal, QC, H3T 1J4, Canada

$10{ }^{2}$ Sebastian Pechmann Research Lab, 66125 Saarbrücken, Germany

11

12

13

14

15

16

17

18

Corresponding Author:

Sebastian Pechmann ${ }^{2}$

Private Address, 66125 Saarbrücken, Germany

Email address: sebastian@pechmannlab.net 
19 Abstract

20

21

22

23

24

25

26

27

Cells are enticingly complex systems. The identification of feedback regulation is critically important for understanding this complexity. Network motifs defined as small graphlets that occur more frequently than expected by chance have revolutionized our understanding of feedback circuits in cellular networks. However, with their definition solely based on statistical overrepresentation, network motifs often lack biological context, which limits their usefulness. Here, we define functional network motifs (FNMs) through the systematic integration of genetic interaction data that directly informs on functional relationships between genes and encoded proteins. Occurring two orders of magnitude less frequently than conventional network motifs, we found FNMs significantly enriched in genes known to be functionally related. Moreover, our comprehensive analyses of FNMs in yeast showed that they are powerful at capturing both known and putative novel regulatory interactions, thus suggesting a promising strategy towards the systematic identification of feedback regulation in biological networks. Many FNMs appeared as excellent candidates for the prioritization of follow-up biochemical characterization, which is a recurring bottleneck in the targeting of complex diseases. More generally, our work highlights a fruitful avenue for integrating and harnessing genomic network data. 
39

40

41

42

\section{Introduction}

Cells are enticingly complex systems (Wolf et al., 2018). Their phenotypic traits often depend on the concerted action of hundreds or even thousands of individual genes and encoded proteins (Lehner, 2013; Boyle et al., 2017). Rationalizing this interdependency is important both for understanding how cellular function is encoded in the genome (Watanabe et al., 2019) as well as the origins of complex diseases (Wray et al., 2018).

The large-scale mapping of protein-protein interactions (PPI) (Bork et al., 2004; Rual et al.,2005; Krogan et al., 2006; Tarassov et al., 2008; Yu et al., 2008) and genetic interactions (GI) (Schuldiner et al., 2005; Costanzo et al., 2016, 2019) has provided a foundation for understanding the functional organization of cellular networks (Barabasi, 2004). Most proteins function through direct contact, i.e. PPIs. Accordingly, clusters of high interconnectivity in PPI networks have revealed strong hierarchical modularity (Han et al., 2004) that ranges from protein complexes (Gavin et al., 2006) to sets of functionally related proteins (Ravasz et al., 2002; Huynen et al., 2003; von Mering et al., 2003). Significant efforts have been undertaken to identify such network modules from the network structure (Park and Bader, 2011) and link them to observable phenotypes (Wang et al., 2012; Vinayagam et al., 2014). In contrast, GIs inform on the functional relationships between genes by quantifying epistatic fitness effects of their deletion mutants (Mani et al., 2008). Normally, GIs are assessed and quantified through the observed growth phenotype of the deletion of gene pairs compared to the expectation based on single deletions of the corresponding genes (Beltrao et al., 2010). Positive GIs are observed when the fitness effect of the double-deletion of both genes of interest is less severe than expected based on the phenotypes of the individual deletions. As an example, the first deletion already disrupts a function that requires both genes/proteins, and the second deletion thus has only a comparably small additional effect. As such, positive GIs are often linked to dependencies within the same function, for example between genes of proteins within a protein complex or linear pathway. Conversely, negative GIs are present when the combined effect of deleting two genes is more severe than expected. Negative GIs are frequently observed between redundant and compensatory processes . However, many GIs have complex and non-trivial origins (Crona et al., 2017; Pirkl et al., 2017; Kuzmin et al., 2018) that await to be further characterized. Similar to 
PPIs, GI networks also display strong hierarchical modularity (Beltrao et al., 2010; Cornish and Markowetz, 2014; Fang et al., 2019) that confers robustness and versatility (Fortuna et al., 2017).

The smallest yet arguably most important recurring unit of modularity in biological networks are network motifs (Milo et al., 2004; Kashtan and Alon, 2005). Network motifs were initially defined as small graphlet architectures of size $\mathrm{k} \approx 3-6$ nodes that occur more frequently than expected by chance, thus assuming that their selection indicates importance (Milo et al., 2004). Enabled by efficient algorithms for their discovery (Kashtan et al., 2004; Schreiber and Schwöbbermeyer, 2005; Wernicke and Rasche, 2006; Przulj, 2006; Kashani et al., 2009), network motifs have dramatically improved our understanding of biological networks. Through their detailed characterization it has become clear that motifs often encode important feedback circuits with distinct functionality (Alon, 2007) such as feed-forward signaling (Goentoro et al., 2009), control of system states (Elowitz and Leibler, 2000), and coordination of decision making (Brandman et al., 2005). In complement, the large-scale analysis of motifs in networks has helped to connect network topology to function (Vázquez et al., 2004), for instance in transcription regulation (Jothi et al., 2009; Wang and Chen, 2010; Song et al., 2017; Teixeira et al., 2017), stress responses (Kim et al., 2012; Hahn et al., 2006), and development (Freeman, 2000).

Importantly, network motifs are primed to encode the evidently pervasive but so far largely elusive feedback and cross-talk pathways within cellular networks required to understand their complexity. However, the usefulness of network motifs to systematically identify regulatory interactions has so far been limited (Konagurthy and Lesk, 2008). Specifically, occurrences of motifs are routinely found in very large numbers, which renders the selection of promising candidates for detailed follow-up characterization challenging. Moreover, despite statistical overrepresentation of their topology most individual network motifs are not evolutionarily conserved (Mazurie et al., 2005), suggesting that only a small fraction of the network motifs may indeed carry function. We therefore hypothesized that an improved identification of network motifs that takes into consideration their individual biological contexts may offer additional insights into the functioning and organization of biological networks. 
100 Here, we defined functional network motifs (FNMs) based on integrating GIs as direct measure 101 of functional relationships between genes into the discovery of motifs in the yeast PPI network. 102 Importantly, only by starting from motifs in the PPI network, FNMs connect to the seminal work

103

104

105

106

107

108

109

110

111

112

113

114

115

116

117

118

119

120

121

122

123

124

125

126

127

128 on the functioning of network motifs (Alon, 2007) and afford to formulate mechanistic hypotheses. This would not be possible to the same extend by starting from GI motifs due to their indirect and complex nature. Remarkably, with occurrences of FNMs about two orders of magnitude less frequent than conventional PPI network motifs, we found FNMs significantly enriched in genes known to be functionally related. Moreover, our comprehensive analyses of FNMs in yeast showed that they are powerful at re-identifying known regulatory interactions as well as discovering interesting new ones. Our work highlights a promising avenue to harness genomic network data towards systematically identifying regulatory interactions and further breaking down biological complexity.

\section{Materials \& Methods}

\section{Data and code availability}

Project data and computer code generated for this study are available at https://www.github.com/pechmannlab/FNM and archived on Zenodo at doi:10.5281/zenodo.5818964.

\section{Data sources}

The Saccharomyces cerevisiae protein interaction network was obtained from BIOGRID release 3.5.185 (Oughtred et al., 2019) and filtered for physical protein interactions. Yeast genetic interactions were retrieved from (Costanzo et al., 2016). The top and bottom 5\% of all pairwise interaction scores were considered significant and used to build a genetic interaction network. The set of curated suppressor interactions from (van Leeuwen et al., 2016) was used as benchmark example of functionally related genes. We further used the annotations of yeast protein complexes from the MIPS database (Güldener et al., 2006), the consensus transcription 
129 factors from YEASTRACT (Teixeira et al., 2017; Monteiro et al., 2020), and the metabolic

130 network model Yeast8 (Lu et al., 2019). Co-expression in response to environmental

131 perturbations was assessed with the expression data from (Gasch et al., 2000).

133 Enumeration of FNMs

134 Network motifs were generated through exhaustive enumeration of graphlets in the S. cerevisiae 135 protein-protein interaction (PPI) network. Our approach followed the algorithm developed by 136 (Kashani et al., 2009) wherein each motif class of numbers of nodes per layer, defined through 137 the integer compositions of the motif size k, was enumerated by standard depth-first-search.

138 Herein, starting from a selected source node, all unique patters of connectivity of a given size 139 were recorded, and all nodes in the yeast PPI network were used as a source node. We 140 considered a graphlet, i.e. a motif in the PPI network, as a 'functional network motif' (FNM) if at 141 least $50 \%$ of all possible non-self genetic interaction edges within the graphlet were present, and 142 the source node had direct genetic interactions with all nodes in the most distant layer. The 143 threshold choice of 50\% was arbitrary but reflected a best trade-off between sufficient selectivity 144 of a high threshold and sufficient remaining motif occurrences for further analysis. All motifs of 145 sizes $\mathrm{k}=3,4,5,6$ were generated.

147 Two deliberate constraints served to improve both computational tractability and biological 148 interpretability. Proteins that interact with hundreds or thousands of other proteins play important roles in the organization of cellular networks but rarely exhibit strong specificity and selectivity. To identify functional network motifs that may be involved in specific feedback circuits rather than network hubs, we only considered proteins of degree $d_{\max }<50$, while thresholds of $d_{\max }<25$ and $\mathrm{d}_{\max }<100$ were also tested. Importantly, the excluding of the most highly connected network hubs made the exhaustive motif enumeration in the yeast PPI computationally feasible up to a motif size of $\mathrm{k}=6$. Moreover, we only considered interactions between protein complexes but not between subunits of the same protein complexes. The rationale for this was that we wanted to identify putative feedback links that connect biological function rather than understand the architecture of protein complexes that often act as functional unit. Network randomizations were performed with the R package BiRewire (Iorio et al., 2016). Motifs were generated for 
159 randomized PPI networks, and randomized genetic interaction networks. 30 randomizations were

160 found to yield sufficiently converging reference values (Figure S1).

161

162 Analysis of FNMs

163 Motifs were clustered by iteratively identifying and merging maximally overlapping individual

164 motifs. A complex-based motif interaction network was generated by considering all yeast

165 genes/proteins from the FNMs and merging protein complex subunit genes into joint nodes.

166 Genes encoding proteins that are part of more than one protein complex were omitted.

167 Transcriptional co-regulation of genes from the same motif was quantified through their average

168 pairwise cosine similarity between the time-course expression changes in response to

169 environmental perturbations (Gasch et al., 2000). All visualization was done in R. 


\section{Results}

172 The identification of regulatory interactions is critically important for understanding cellular

173 complexity. Network motifs have made a foundational impact on our understanding of the

174 regulation of biological networks. Because PPIs and GIs are generally not correlated, the

175 integration of GI data provides a premier opportunity to dramatically expand the functional

176 analysis of network motifs. We here hypothesized that an expanded definition of network motifs

177 based on both PPIs as record of protein activity as well as GIs that quantify functional

178 relationships should improve their usefulness for biological discovery. To test this idea, we

179 defined functional network motifs (FNMs) as graphlets in the yeast PPI network that were also

180 enriched in GIs. Specifically, for a graphlet to be considered an FNM we required the presence of

181 GIs between the source node and all nodes in the most distant layer, i.e. GIs that span the full

182 graphlet, as well as overall in $\geq 50 \%$ of all possible non-self edges (Figure 1A). The threshold

183 of 50\% was arbitrary but reflected our best choice in a trade-off between stringency and sufficient

184 motif occurrences that remained for further analysis (Figure S1A).

Ultimately, the causality of feedback and regulatory interactions can only be established through experimental perturbation and validation. Our aim here was to provide a computational strategy for the identification of select high-confidence candidates out of the very large number of possible network motifs for future characterization and validation. We here considered as PPI network motifs the collection of all occurrences, i.e. specific sets of genes and encoded proteins, whose wiring architectures were observed at frequencies greater than chance in the yeast PPI. Similarly, FNMs were defined to describe the specific sets of genes and encoded proteins that were found in PPI motifs filtered for GI content.

We implemented an algorithm for the efficient and exhaustive enumeration of all motifs of size $\mathrm{k}=3,4,5,6$ in the yeast PPI network (see Methods). Two deliberate constraints were imposed to reduce computational cost while increasing biological interpretability. Because the most highly connected nodes in the yeast PPI are usually protein interaction hubs rather than specific regulators, we omitted the highest degree nodes from the network. Moreover, we only considered at most one protein complex subunit per motif as we were interested in links between functional 
201 units rather than the architecture of protein complexes. Remarkably, we counted about two

202 orders of magnitude fewer FNMs than PPI motifs independent of motif size (Figure 1B). While

203 the exact numbers depended on the choice of the threshold, in all cases GIs exerted strong

204 selectivity on the sets of genes and encoded proteins connected within FNMs. For comparison,

205 we generated all PPI motifs in the yeast PPI and randomized PPI networks as well as FNMs

206 upon randomization of the GI network (Figure S1B, see Methods). The obtained results

207 followed expected trends. Illustrated for the most populated topologies, motifs occurred more

208 frequently in the PPI network than in randomized PPI networks, thus could also be considered

209 classical network motifs (Figure 1C). Similarly, FNMs were substantially more frequent than in

210 randomized GI networks, suggesting that the selection of motifs based on high GI density is

211 selective and meaningful (Figure 1C, S1C,D). Taken together, FNMs collectively identify the

212 same topologies as PPI network motifs but their individual occurrences were substantially more

213 rare.

214

215

216

217 FNMs are enriched in functionally related genes

218 Naturally, a more selective definition of network motifs resulted in lower counts. To test whether

219 our definition of FNMs was indeed meaningful, we evaluated if they preferentially contained

220 functionally related and important genes. One intriguing example of functionally related genes is

221 given by genetic suppressors, pairs of genes where the phenotypic effect of a mutation in one

222

223

gene is compensated by the other. Importantly, while yeast suppressors genetically interact (van

224

Leeuwen et al., 2016), a single GI alone would not bias a motif towards an FNM (Figure 2A).

225

Strikingly, we found a significant enrichment of suppressor interactions in FNMs compared to

226

conventional PPI network motifs ( $\mathrm{p}<10^{-16}$, Fisher's Exact Test, Figure 2A). Thus, FNMs were directly enriched in functional interactions.

227

228 Further support for our definition of FNMs was drawn from an analysis of essential genes and genes that code for protein complex subunits. Essential genes are functionally indispensable and their deletion mutants are by definition not viable. However, GIs of essential genes can be 
231 measured for instance with DAmP alleles that perturb their expression, and are often

232 pronounced. Similarly, protein complex subunits are important due to the functional importance

233 of the complex as well as the high fitness cost of mutants in individual subunits that can

234 destabilize the whole complex. We found FNMs to contain a higher fraction of both essential

235 genes ( $<<10^{-16}$, Wilcoxon-Mann-Whitney (WMW) test, Figure 2B) and genes coding for

236 protein complex subunits $\left(\mathrm{p}<10^{-16}\right.$, WMW test, Figure 2B) than PPI network motifs.

237

238 To estimate redundancy in the large number of FNMs, we next clustered them based on node 239 overlap (see Methods). We found that $93.6 \%$, i.e. the vast majority of FNMs concentrated in 240 clusters of sizes $\approx 7-70$, while 26078 FNMs did not cluster (Figure 2C). Of note, very few 241 genes were found responsible for this agglomeration of FNMs. While only 6.4\% of FNMs did 242 not cluster, they represented $87.7 \%$ of all genes and proteins found in FNMs (Figure 2C). The 243 genes in clustered FNMs had a significantly higher representation in different motifs compared to genes only found in non-clustered FNMs $\left(\mathrm{p}=5.3 \cdot 10^{-134}\right.$, WMW test, Figure 2D) because they

245 also had on average a significantly higher degree $\left(\mathrm{p}=2.3 \cdot 10^{-30}\right.$, WMW test, Figure 2D).

246 Importantly, the non-clustered FNMs retained their enrichment in suppressor interactions,

247 essential genes and complex subunits. Thus, FNMs defined through integration of PPIs and GIs

248 are indeed representing collections of important functional interactions. While clusters of FNMs

249 share strong similarity to larger modules in the PPI network, the FNMs that do not cluster should 250 be particularly interesting.

Genetic interactions in FNMs are orthogonal to protein interactions

253 Having established that FNMs indeed reflect known functional dependencies between genes, we 254 next sought to better understand the composition of their genetic interactions. Overall, negative GIs were clearly more prevalent in FNMs (Figure 3A) but we could not identify any trends that link subsets of FNMs to predominantly positive or negative GIs. This was not unexpected as GIs are known to be complex and strongly context dependent (Markowetz et al., 2007; Kuzmin et al., 258 2018). 
260 To test any consistency and correlation of GIs and PPIs, we generated a protein complex

261 interaction network by considering all interactions contained in the non-clustering FNMs and

262 mapping them onto joint nodes for the annotated yeast protein complexes. Protein complexes are

263 a good test case because they form important functional units but may interact through multiple

264 subunits. For each pairwise interaction between two complexes, we collected all GIs contained in

265 the corresponding FNMs. While these included both repeated occurrences of the same GI edge

266 from different FNMs and GIs between different complex subunits, almost all pairs of complexes

267 had multiple different GIs. Remarkably, the consensus GIs between protein complexes extracted

268 from the FNMs showed almost perfect consistency towards negative or positive interactions

\section{9 (Figure 3B, S2).}

270

271

The full yeast protein complex interaction map highlighted important functional connections as well as the orthogonal nature of PPIs and GIs (Figure 3C). Some of the most central protein machines required for genome maintenance such as DNA polymerases dominated the observed negative GIs. Complexes with positive GIs included examples of protein targeting and translocation such as the ESCRT complexes involved in endosomal sorting or the Vam3/Vam7 complex that functions in vacuolar trafficking. Only some complexes shared both PPIs and GIs. Notably, only $25.9 \%$ of protein complex pairs that interacted physically also interacted genetically (Figure 3D). In turn, 29.8\% of edges between protein complexes were only PPIs, while $44.3 \%$ GIs (Figure 3D). Thus, the GIs captured by the FNMs were both consistent and largely orthogonal to the PPIs.

In the extreme, an FNM of size $\mathrm{k}=6$ can connect six different protein complexes, for instance complexes that function in DNA replication and repair (Figure 3E). In this exemplary FNM, SLD5 of the GINS complex that is important for DNA replication connected with both PPIs and negative GIs to DPB3 of the DNA polymerase epsilon subunit, DPB11 as scaffolding protein of the Dpb11p/Sld2p complex important for DNA replication initiation, and TOF1 of the replication pausing checkpoint complex. Further connections were observed from DPB11 to MMS4 of the Holiday-junction-resolvase that functions in recombination and DNA repair, as well as a PPI and positive GI between DBF4, which is the regulatory subunit of a kinase 
290

291

292

293

294

295

296

297

298

299

300

301

302

303

304

305

306

307

308

309

310

311

312

313

314

315

316

317

318

complex important for the initiation of DNA replication, and TOF1. The interplay between the regulation of DNA replication and repair is in general well established and this motif was just one example highlighting the potential of FNMs to identify strong candidates for regulatory interactions from genomic network data.

\section{FNMs identify strong candidates for feedback regulation}

Having shown that FNMs capture consistent and meaningful interactions, we next sought to test the potential of FNMs to identify putative regulatory or cross-talk motifs. While some protein complexes come into direct contact, others only communicate through additional proteins with auxiliary roles such as sensing, regulation, or signaling. The betweenness centrality is an established network metric that quantifies the relative fraction of shortest paths that transverse through a node thus indicating its importance for instance for the flow of information across a network. Using the protein complex network generated from merging the FNM interactions of protein complex subunits into joint nodes, we therefore computed the betweenness centrality for all auxiliary nodes, i.e. non-complex nodes, as defined by the shortest paths between all pairs of protein complexes (Figure 4A).

To test whether the betweenness centrality of the auxiliary nodes identified by the FNMs differed, the corresponding betweenness centralities were also computed for all proteins in the yeast PPI network filtered at maximum degree $d_{\max }<50$, the network used to generate the FNMs. Moreover, we also computed as a control betweenness centrality values for all auxiliary nodes in the full yeast PPI network. Betweenness centrality values were significantly higher for FNMs compared to the filtered PPI network ( $\mathrm{p}=1.06 \cdot 10^{-6}$, WMW test, Figure 4B), and significantly higher for the filtered PPI network than for the full PPI network $\left(p=9.24 \cdot 10^{-174}\right.$, WMW test, Figure 4B). In part this observation scaled with the corresponding network sizes wherein the shortest paths between protein complexes concentrated to fewer nodes in smaller networks (Figure 4D). However, considering only contributing nodes with betweenness centrality values $>0$ suggested that the differences in betweenness centralities for the filtered and full PPI networks was independent of the effective network size and rather a function of the presence of 
319 very highly connected network hubs (Figure 4D). The higher betweenness centrality for the

320 FNM network must be the result of both a smaller network and the lack of hubs.

321

322 The investigation of the the auxiliary nodes with the highest betweenness centrality values in the

323

324

325

326

327

328

329

330

331

332

333

334

335

336

337

338

339

340

341

342

343

344

345

346

347

348

FNM network lead to several proteins known for their regulatory or cross-talk roles (Figure $\mathbf{4 E}$ ). BOL1 is a mitochondrial matrix protein and assembly factor involved in the transfer of [4Fe-4S] clusters from the ISA complex to client proteins (Uzarska et al., 2016). TOM71 is assumed to aid in the targeting of proteins to mitochondria (Schlossmann et al., 1996). TAH1 is a HSP90 chaperone co-factor required for C/D small nucleolar ribonucleoprotein assembly (Jiméenez et al., 2012). The mitochondrial thioredoxin peroxidase PRX1 is important for sensing and countering oxidative stress (Pedrajas et al., 2000). And the membrane transporter PDR15 contributes to the cross-talk between stress response and the pleiotropic drug resistance network for cellular detoxification (Wolfger et al., 2004).

To explore potentially novel regulatory interactions in a more controlled test scenario, we next identified all FNMs that connect two of the most important and best-characterized subnetworks in the cell, namely the set of transcription factors (TFs) forming the transcription regulatory network, and the yeast metabolic network. The metabolic network engages in extensive crosstalk to maintain overall energy homeostasis, predominantly through reporter metabolites that bind to regulatory proteins such as transcription factors or metabolic enzymes, but also mediated through PPIs (Grüning et al., 2010). Remarkably, we only found 11 FNMs or groups of interlinked FNMs that connect these large and important systems, each with distinct patters of PPIs and GIs between TFs and metabolic enzymes (Figure 4E).

The FNMs included the well characterized response to glucose repression where the TF MIG1 directly inhibits the expression of the sucrose hydrolyzing enzyme SUC2. Both were additionally found to genetically interact with the pre-mRNA splicing machinery. The global regulator of respiratory gene expression HAP3 was linked to the enzyme ALG9 involved in N-linked glycosylation in the endoplasmic reticulum via two sensors of alkaline pH, RIM8 and RIM21, as well as USA1, the scaffold protein of the ubiquitin ligase HRD1. It is plausible to speculate about 
349 a feedback circuit that involves pH sensing and selective degradation. Similarly, the specialized

350 proteasome and stress response TF RPN4 was found to interact not only with the core

351 proteasome subunit PRE7, but via the E3 ligases UBR1 and UBR2 as well as the E2 RAD6 also

352 with the metabolic enzyme DFR1 involved in tetrahydrofolate biosynthesis within respiratory

353 metabolism. RAD6 is known to form complexes with UBR1 and UBR2 respectively with

354 different substrate specificities, thus may well contribute to the coordination of RPN4 and DFR1

355 protein levels. As such, this FNM appeared as an exceptionally strong candidate for contributing

356 to the coordination of transcription regulation and metabolism under stress. In summary, the

357 FNMs have revealed very promising candidates for putative regulatory interactions in low

358 enough numbers that render them immediately amenable for biochemical characterization.

\section{Transcriptionally co-regulated FNMs are rare but important}

361

362

363

364

365

366

367

368

369

370

371

372

373

374

375

376

377

378

Finally, cellular systems are highly dynamic and constantly rewire their networks (Pe'er et al., 2001), complexes (de Lichtenberg et al., 2005), and also network motifs (Prill et al., 2005; Doyle and Csete, 2005) under changing conditions. For instance, dynamically adapting motifs temporally coordinate the global transcription regulation of metabolism (Chechik et al., 2008). Without knowledge of the network states under perturbation, the inference of dynamically changing network motifs and their activity under different conditions is challenging and at most approximate. However, the identification of strongly co-regulated motifs offered an indication of which motifs retain their activity under changing conditions.

Specifically, by analyzing the transcriptional responses to environmental perturbations we found that co-regulated FNM were rare (Figure 5A). In fact, less than $1 \%$ of FNMs were what can be considered very strongly co-regulated, and about 5\% as strongly co-regulated (Figure 5B).

Moreover, while smaller motifs of size $\mathrm{k}=3$ naturally had higher fraction of strongly coregulated FNMs, co-regulation was else not biased by motif size (Figure 5C).

Some of the co-regulated FNMs were interlinked and clustered into well-characterized modules, for example the core RNA splicing machinery that was found systematically co-regulated in response to several stress conditions (Figure 5D). But the small individual FNMs highlighted the 
379

380

381

382

383

384

385

386

387

388

389

390

391

392

393

394

395

396

397

398

399

400

401

402

403

404

405

406

407

408

more interesting connections. For instance, the nuclear and exosome-associated RNA binding protein MPP6, involved in pre-mRNA surveillance, linked through PPIs to both AIR2, an RNAbinding protein of the TRAMP nuclear RNA surveillance complex, and the non-catalytic exosome core component RRP4 (Figure 5E). Moreover, RRP4 was interacting through negative GIs with both MPP6 and AIR2, underlining substantial cross-talk between RNA surveillance and quality control (Lisbeth-Carolina et al., 2020).

Last, many motifs likely alter their activity under changing conditions. For instance, the previously identified FNM of the stress response TF RPN4 that is linked by an E2/E3 ligase system to the metabolic enzyme DFR1 showed a distinct pattern of expression changes. RPN4 as well as the direct TF target PRE7 were strongly induced under heat stress (Figure 5F). In turn, the RPN4-facing E3 UBR2 was initially mildly down-regulated, but overall the expression of the E2 and E3 ligases did not change while DFR1 was strongly down-regulated to no longer detected (Figure 5F). It is plausible to speculate that this FNM contributes to the coupling of transcription and protein levels in adaptation to heat stress by shifting the protein degradation activity to the RAD6-UBR1 complex for the degradation of DFR1. Of note, while UBR1 is normally nuclear, it readily becomes cytosolic under stress (Breker et al., 2013) where it could target DFR1 and other proteins to maintain cellular homeostasis. FNMs are no finitive blueprint of cellular regulation, but offer a most promising glimpse into putative regulatory interactions from already available genomic data that warrant further investigation.

\section{Discussion}

Network motifs have revolutionized our understanding of feedback circuits and the organization of cellular networks. However, solely defined through statistical over-representation they often lack biological context. Here, we have defined functional network motifs, or FNMs, through the integration of genetic interaction data that directly informs on functional relationships between genes and encoded proteins. By capturing known and novel regulatory interactions, FNMs have

been found a very promising strategy towards the systematic identification of feedback and cross-talk in cellular regulation. Importantly, the description as 'functional' is intended as 
409

410

411

412

413

414

415

416

417

418

419

420

421

422

423

424

425

426

427

428

429

430

431

432

433

434

435

436

437

categorical rather than definite. Any notion of biological function itself is strongly contextdependent and subject to debate (Keeling et al., 2019). More opportunities await for further improving any definition of FNMs.

Ultimately, causation can only be established through targeted perturbations. Large-scale perturbation studies (McIsaac et al., 2012; Hackett et al., 2016; Caldera et al., 2019; Hackett et al., 2020) offer a promising outlook. However, even in absence of control perturbations, additional information, especially the directionality of interactions (Vinayagam et al., 2014), can be very informative as it allows to explore functional parameter ranges through the modeling of motif dynamics. As more genomic data are generated under non-standard laboratory conditions, it will certainly become easier to dissect the intricacies of cellular regulation.

Already now, FNMs offer many interesting insights and a strategy through the motif-based integration of omics data that is readily extendable to additional data types. Support for this rationale is given by the GIs observed in the FNMs. Similar to other genome-scale measurables, GIs are inherently noisy. Consequently, GIs are routinely analyzed through correlations of their genomic interaction profiles rather than as individual interactions (VanderSluis et al., 2018). Through small clusters of GIs between functionally related genes, the FNMs achieve more confidence than would be offered by single GIs but are less generic than genome-wide profiles.

To this end, regulatory events in the cell extend far beyond physical interactions between macromolecules as captured by PPI networks. Signaling can involve feedback through temporal (Harrigan et al., 2018) and spatial organization (Santos et al., 2012), the dynamic activation and deactivation through modification (Hirano et al., 2016), and even the folding of proteins and RNA (Rutherford and Zuker, 1994). Moreover, some systems are truly determined by collective behavior that can only be understood at a systems level. For instance, the cellular protein folding capacity balances the proteome with available quality control pathways (Draceni and Pechmann, 2019) wherein the competition for the shared folding capacity is governed through differential interaction specificities (Pechmann, 2000). Central to many aspects of cell integrity, protein 
438 homeostasis is a particularly important example where integrative approaches have been very 439 promising with a lot of open opportunity (Rizzolo et al., 2017).

440

441 An orthogonal approach to the discovery of critical regulators follows recent advances in control 442 and systems theory to identify the controlling nodes of networks from their topology (Liu et al., 443 2011; Zanudo Tejeda et al., 2017) and even dynamics (Baggio et al., 2021). Such avenues 444 currently remain challenging for genome-scale networks, predominantly due to strong 445 assumptions as well as insufficient and incomplete data, but will most certainly continue to gain 446 in importance. Even stronger poised will be the synthesis of these ideas from genomics, systems 447 biology, and control. Due to their simplicity, FNMs can immediately contribute to the systematic 448 identification of cross-talk and regulatory interactions. Iterative cycles of improvement will 449 continue to refine the prioritization of genes for follow-up characterization (Kuang et al., 2020), 450 decipher complex regulatory logic (Buchler et al., 2003), and support the re-engineering of 451 critical protein sense-response systems (Glasgow et al., 2020). 


\section{References}

457 Alon U. 2007. Network motifs: theory and experimental approaches. Nat Rev Genet 8(6):450-61. 458

459

460

461

462

463

464

465

466

467

468

469

470

471

472

473

474

475

476

477

478

479

480

481

482

483

484
Baggio G, Bassett DS, and Pasqualetti F. 2021. Data-driven control of complex networks. Nat Comms 12(1):1429.

Barabasi A. 2004. NETWORK BIOLOGY: UNDERSTANDING THE CELL'S FUNCTIONAL ORGANIZATION. Net Rev Genet 5:101-114.

Beltrao P, Cagney G, and Krogan NJ. 2010. Quantitative genetic interactions reveal biological modularity. Cell 141(5):739-745.

Bork P, Jensen LJ, von Mering C, Ramani AK, Lee I, and Marcotte EM. 2004. Protein interaction networks from yeast to human. Curr Opin Struct Biol 14(3):292-299.

Boyle EA, Li YI, and Pritchard JK. 2017. An Expanded View of Complex Traits: From Polygenic to Omnigenic. Cell 169(7):1177-1186.

Brandman O, Ferrell JJ, Li R, and Meyer T. 2005. Interlinked fast and slow positive feedback loops drive reliable cell decisions. Science 310(5747):496-8.

Breker M, Gymrek M, Moldavski O, and Schuldiner M. 2013. LoQAtE Localization and Quantitation ATlas of the yeast proteomE. A new tool for multiparametric disscection of singleprotein behavoir in response to biological perturbations in yeast. Nucl Acids Res 42(D1):D726D730.

Buchler NE, Gerland U, and Hwa T. 2003. On schemes of combinatorial transcription logic. Proc Acad Natl Sci 100(9):5136-5141. 
485 486 487

488 489 490

491

492 493 494

495 496 497 498 499 500 501 502 503 504 505 506 507 508 509

Caldera M, Müller F, Kaltenbrunner I, Licciardello MP, Lardeau CH, Kubicek S, and Menche J. 2019. Mapping the perturbome network of cellular perturbations. Nat Comms 10:5140.

Chechik G, Oh E, Rando O, Weissman J, Regev A, and Koller D. 2008. Activity motifs reveal principles of timing in transcriptional control of the yeast metabolic network. Nat Biotechnol 26(11):1251-9.

Cornish AJ and Markowetz F. 2014. SANTA: Quantifying the Functional Content of Molecular Networks. PLoS Comput Biol 10(9):e1003808.

Costanzo M, Kuzmin E, van Leeuwen J, Mair B, Moffat J, Boone C, and Andrews B. 2019. Global Genetic Network and the Genotype-to-Phenotype Relationship. Cell 177:85-97.

Costanzo M, VanderSluis B, Koch EN, Baryshnikova A, Pons C, Tan G, Wang W, Usaj M, Hanchard J, Lee SD, et al.. 2016. A global genetic interaction network maps a wiring diagram of cellular function. Science 353(6306):aaf1420-1.

Crona K, Gavryushkin A, Greene D, and Beerenwinkel N. 2017. Inferring genetic interactions from comparative fitness data. eLife 6:e28629.

de Lichtenberg U, Jensen LJ, Brunak S, and Bork P. 2005. Dynamic complex formation during the yeast cell cycle. Science 307(5710):724-727.

Doyle J and Csete M. 2005. Motifs, Control, and Stability. PLoS Biol 3(11):e392.

Draceni Y and Pechmann S. 2019. Pervasive convergent evolution and extreme phenotypes define chaperone requirements of protein homeostasis. Proc Natl Acad Sci 116:20009-20014.

Elowitz M and Leibler S. 2000. A synthetic oscillatory network of transcriptional regulators. Nature 403(6767):335-8. 
516 Fang G, Wang W, Paunic V, Heydari H, Costanzo M, Liu X, Liu X, VanderSluis B, Oately B, 517 Steinbach M, et al.. 2019. Discovering genetic interactions bridging pathways in genome-wide 518 association studies. Nat Comms 10:4272.

519

520 Fortuna MA, Zaman L, Ofria C, and Wagner A. 2017. The genotype-phenotype map of an 521 evolving digital organism. PLoS Comput Biol 13(2):e1005414.

522

523

Freeman M. 2000. Feedback control of intercellular signalling in development. Nature 524 408(6810):313-9.

525

526

Gasch A, Spellman P, Kao C, Carmel-Harel O, Eisen M, Storz G, Botstein D, and Brown P. 527 2000. Genomic expression programs in the response of yeast cells to enrivonmental changes. 528 Mol Biol Cell 11(12):4241-57.

529

530

Gavin A, Aloy P, Grandi P, Krause R, Boesche M, Marzioch M, Rau C, Jensen L, Bastuck S, 531 Dümpelfeld B, et al.. 2006. Proteome survey reveals modularity of the yeast cell machinery. Nature 440(7084):631-636.

533

534

Glasgow AA, Huang YM, Mandell DJ, Thompson M, Ritterson R, Loshbaugh AL, Pellegrino J, 535 Krivacic C, Pache RA, Barlow KA, et al.. 2020. Computational design of a modular protein 536 sense-response system. Science 366(6468):1024-1028.

537

538 Goentoro L, Shoval O, Kirschner MW, and Alon U. 2009. The Incoherent Feedforward Loop 539 Can Provide Fold-Change Detection in Gene Regulation. Mol Cell 36:894-899.

541 Grüning NM, Lehrach H, and Ralser M. 2010. Regulatory crosstalk of the metabolic network. 542 Trends Biochem Sci 35(4):220-227. 543 
544 Güldener U, Münsterkötter M, Oesterheld M, Pagel P, Ruepp A, Mewes HW, and Stümflen V.

545 2006. MPact: the MIPS protein interaction resource on yeast. Nucl Acids Res 34:D436-41.

546

547 Hackett SR, Baltz EA, Coram M, Wranik BJ, Kim G, Baker A, Fan M, Hendrickson DG, Berndl

548 M, and McIsaac RS, et al.. 2020. Learning causal networks using inducible transcription factors

549 and transcriptome-wide time series. Mol Syst Biol 16:e9174.

550

551 Hackett SR, Zanotelli VRT, Xu W, Goya J, Park JO, Perlman DH, Gibney PA, Botstein D,

552 Storey JD, and Rabinowitz JD, et al.. 2016. Systems-level analysis of mechanisms regulating

553 yeast metabolic flux. Science 354(6311):aaf2786.

554

555

556

Hahn JS, Neef DW, and Thiele DJ. 2006. A stress regulatory network for co-ordinated activation of proteasome expression mediated by yeast heat shock transcription factor. Mol Microbiol 557 60(1):240-251.

558

559

Han JDJ, Bertin N, Hao T, Goldberg DS, Berriz GF, Zhang LV, Dupuy D, Walhout AJM,

560

Cusick ME, Roth FP, et al.. 2004. Evidence for Dynamically Organized Modularity in the Yeast

561

Protein-Protein Interaction Network. Nature 430(6995):88-93.

562

563

Harrigan P, Madhani HD, and El-Samad H. 2018. Real-Time Genetic Compensation Defines the 564 Dynamic Demands of Feedback Control. Cell 175(3):877-886.

565

566

Hirano A, Fu YH, and Ptacek LJ. 2016. The intricate dance of post-translational modifications in 567 the rhythm of life. Nat Struct Mol Biol 23(12):1053-1060.

568

569

Huynen MA, Snel B, von Mering C, and Bork P. 2003. Function prediction and protein

570 networks. Curr Opin Cell Biol 15(2):191-198. 
572 Iorio F, Bernardo-Faura M, Gobbi A, Cokelaer T, Jurman G, and Saez-Rodriguez J. 2016.

573 Efficient randomization of biological networks while preserving functional characterization of

574 individual nodes. BMC Bioinformatics 17:542.

575

576 Jiméenez B, Ugwu F, Zhao R, Ortí L, Makhnevych T, Pineda-Lucena A, and Houry WA. 2012.

577 Structure of minimal tetratricopeptide repeat domain protein Tah1 reveals mechanism of its

578 interaction with Pih1 and Hsp90. J Biol Chem 287(8):5698-709.

579

580

Jothi R, Balaji S, Wuster A, Grochow JA, Gsponer J, Przytycka TM, Aravind L, and Babu MM.

581

2009. Genomic analysis reveals a tight link between transcription factor dynamics and regulatory

582

network architecture. Mol Syst Biol 5:294.

583

584

Kashani ZRM, Ahrabian H, Elahi E, Nowzari-Dalini A, Ansari ES, Asadi S, Mohammadi S,

585

Schreiber F, and Masoudi-Nejad A. 2009. Kavosh: a new algorith for finding network motifs.

586

BMC Bioinformatics 10:318.

587

588

Kashtan N and Alon U. 2005. Spontaneous evolution of modularity and network motifs. Proc

589

Natl Acad Sci 102(39):13773-13778.

590

591

Kashtan N, Itzkovitz S, Milo R, and Alon U. 2004. Efficient sampling algorithm for estimating

592 subgraph concentrations and detecting network motifs. Bioinformatics 20(11):1746-1758.

593

594

Keeling DM, Garza P, Nartey CM, and Carvunis AR. 2019. The meanings of 'function' in biology and the problematic case of de novo gene emergence. eLife 8:e47014.

596

597 Kim D, Kim MS, and Cho KH. 2012. The core regulation module of stress-responsive regulatory 598 networks in yeast. Nucl Acids Res 40(18):8793-8802.

599

600 Konagurthy AS and Lesk AM. 2008. On the origin of distribution patterns of motifs in biological 601 networks. BMC Syst Biol 2:73. 
602

603 Krogan N, Cagney G, Yu H, Zhong G, Guo x, Ignatchenko A, Li J, Pu S, Datta N, Tikuisis A, et 604 al.. 2006. Global landscape of protein complexes in the yeast Saccharomyces cerevisiae. Nature 605 440(7084):637-634.

606

607

608

Kuang D, Truty R, Weile J, Johnson B, Nykamp K, Araya C, Nussbaum RL, and Roth FP. 2020.

Prioritizing genes for systematic variant effect mapping. Bioinformatics 36(22-23):5448-5455.

609

610

Kuzmin E, VanderSluis B, Wan W, Tan G, Deshpande R, Chen Y, Usaj M, Balint A, Usaj MM, 611 van Leeuwen J, et al.. 2018. Systematic analysis of complex genetic interactions. Science 612 360:eaao1729.

613

614 Lehner B. 2013. Genotype to phenotype: lessons from model organisms for human genetics. Nat 615 Rev Genet 14(3):168-178.

616

617 Lisbeth-Carolina A, Paul B, Reiter T, Gendron L, Rajan AAN, Montpetit R, Trahan C, 618 Pechmann S, Oeffinger M, and Montpetit B, et al.. 2020. Altered rRNA processing disrupts 619 620 nulcear RNA homeostasis via competition for the poly(A)-binding protein Nab2. Nucl Acids Res 48(20):11675-11694.

621

Liu YY, Slotine JJ, and Barabási AL. 2011. Controllability of complex networks. Nature 473(7346):167-73.

624

Lu H, Li F, Sanchez B, Zhu Z, Li G, Domenzain I, Marcisauskas S, Anton P, D L, Lieven C, et al.. 2019. A consensus S. cerevisiae metabolic model Yeast8 and its ecosystem for comprehensively probing cellular metabolism. Nat Commun 10(1):3586.

628

Mani R, St Onge RP, Hartman 4th JL, Giaever G, and Roth FP. 2008. Defining Genetic 630 Interactions. Proc Natl Acad Sci 105(9):3461-6. 
632 Markowetz F, Kostka D, Troyanskaya OG, and Spang R. 2007. Nested effects models for high633 dimensional phenotyping screens. Bioinformatics 23:i305-i312.

634

635 Mazurie A, Bottani S, and Vergassola M. 2005. An evolutionary and functional assessment of 636 regulatory network motifs. Genome Biol 6:R35.

637

638

McIsaac RS, Petti AA, Bussemaker HJ, and Botstein D. 2012. Perturbation-based analysis and 639 modeling of combinatorial regulation in the yeast sulfur assimilation pathway. Mol Biol Cell

640 23:2993-3007.

641

642

Milo R, Itzkovitz S, Kashtan N, Levitt R, Shen-Orr S, Ayzenshtat I, Sheffer M, and Alon U. 643 2004. Superfamilies of Evolved and Designed Networks. Science 303(5663):1538-42.

Monteiro PT, Oliveira J, Pais P, Antunes M, Palma M, Cavalheiro M, Galocha M, Godinho CP, 646 Martins LC, Bourbon N, et al.. 2020. YEASTRACT+: a portal for cross-species comparative 647 genomics of transcription regulation in yeasts. Nucl Acics Res 48:D642-D649.

Oughtred R, Stark C, Breitkreutz BJ, Rust J, Boucher L, Chang C, Kolas N, O’Donnell L, Leung G, McAdam R, et al.. 2019. The bioGRID interaction database: 2019 update. Nucl Acids Res 47(D1):D529-D541.

Park Y and Bader JS. 2011. Resolving the structure of interactomes with hierarchical agglomerative clustering. BMC Bioinformatics 12:S44.

655

Pechmann S. 2000. Programmed trade-offs in protein folding networks. Structure 28:1361-1375. of Saccharomyces cerevisiae contain one conserved cystein type peroxiredoxin with thioredoxin 660 peroxidase activity. J Biol Chem 275(21):16296-301. 
662 Pe'er D, Regev A, Elidan G, and Friedman N. 2001. Inferring subnetworks from perturbed

663 expression profiles. Bioinformatics 17:S215-S224.

664

665 Pirkl M, Diekmann M, van der Wees M, Beerenwinkel N, Fröhlich H, and Markowetz F. 2017.

666 Inferring modulators of genetic interactions with epistatic nested effect models. PLoS Comput

667 Biol 13(4):e1005496.

668

669

670

Prill RJ, Iglesias PA, and Levchenko A. 2005. Dynamic Properties of Network Motifs Contribute to Biological Network Organization. PLoS Biol 3(11):e343.

671

672

Przulj N. 2006. Biological network comparison using graphlet degree distribution.

673 Bioinformatics 23:e177-e183.

674

675

Ravasz E, Somera A, Mongru D, Oltvai Z, and Barabasi A. 2002. Hierarchical Organization of Modularity in Metabilic Networks. Science 297:1551-1555.

677

678

Rizzolo K, Huen J, Kumar A, Phanse S, Vlasblom J, Kakihara Y, Zeineddine HA, Minic Z,

Snidder J, Wang W, et al.. 2017. Features of the Chaperone Cellular Network Revealed through

Systematic Interaction Mapping. Cell Rep 20:2735-2748.

681

682

683

Rual J, Venkatesan K, Hao T, Hirozane-Kishikawa T, Dricot A, Li N, Berriz G, Gibbons F, Dreze M, Ayivi-Guedehoussou N, et al.. 2005. Towards a proteome-scale map of the human 684 protein-protein interaction network. Nature 437:1173-1178.

685

686

Rutherford S and Zuker C. 1994. Protein folding and the regulation of signaling pathways. Cell 687 79(7):1129-1132.

688

689 Santos SD, Wollman R, Meyer T, and Ferrell Jr JE. 2012. Spatial Positive Feedback at the Onset 690 of Mitosis. Cell 149(7):1500-1513.

691 
692 Schlossmann J, Lill R, Neupert W, and Court D. 1996. Tom71, a novel homologue of the 693 mitochondrial preprotein receptor Tom70. J Biol Chem 271(30):17890-5.

694

695 Schreiber F and Schwöbbermeyer. 2005. MAVisto: a tool for the exploration of network motifs. 696 Bioinformatics 21(17):3572-3574.

697

698

Schuldiner M, Collins SR, Thompson NJ, Denic V, Bhamidipati A, Punna T, Ihmels J, Andrews 699 B, Boone C, Greenblatt JF, et al.. 2005. Exploration of the function and organization of the yeast 700 early secretory pathway through an epistatic miniarray profile. Cell 123(3):507-519.

701

702

Song Q, Grene R, Heath LS, and Li S. 2017. Identification of regulatory modules in genome 703 scale transcription regulatory networks. BMC Systems Biology 11:140.

704

705

Tarassov K, Messier V, Landry CR, Radinovic S, Serna Molina MM, Shames I, Malitskaya Y, 706 Vogel J, Bussey H, and Michnick SW, et al.. 2008. An in Vivo Map of the Yeast Protein 707 Interactome. Science 320(5882):1465-1470.

708

709

Teixeira MC, Monteiro PT, Palma M, Costa C, Godinho CP, Pais P, Cavalheiro M, Antunes M, 710 Lemos A, Pedreira T, et al.. 2017. YEASTRACT: an upgraded database for the analysis of 711 transcription regulatory networks in Saccharomyces cerevisiae. Nucl Acids Res 46:D348-D353.

712

713

Uzarska MA, Veronica N, Weiler BD, Spantgar F, Ciofi-Baffoni S, Saviello MR, Leonardo G, Mühlenhoff U, Banci L, and Lill R, et al.. 2016. Mitochondrial Bol1 and Bol3 function as assembly factors for specific iron-sulfur proteins. eLife 5:e16673.

716

717

van Leeuwen J, Pons C, Mellor JC, Yamaguchi TN, Friesen H, Koschwanez J, Usaj MM,

718 Pechlaner M, Takar M, Usaj M, et al.. 2016. Exploring genetic suppression interactions on a 719 global scale. Science 354(6312):aag0839.

720 
721 VanderSluis B, Costanzo M, Billmann M, Ward HN, Myers CL, Andrews BJ, and Boone C.

722 2018. Integrating genetic and protein-protein interaction networks maps a functional wiring

723 diagram of a cell. Curr Opin Microbiol 45:170-179.

724

725

Vázquez A, Dobrin R, Sergi D, Eckmann JP, Oltvai ZN, and Barabási AL. 2004. The topological

726

relationship between the large-scale attributes and local interaction patterns of complex

727

networks. Proc Acad Natl Sci 101(52):17940-17945.

728

729

Vinayagam A, Zirin J, Roesel C, Hu Y, Yilmazel B, Samsonova AA, Neumüller RA, Mohr SE,

730

and Perrimon N. 2014. Integrating protein-protein interaction networks with phenotypes reveals

731

signs of interactions. Nat Methods 11(1):94-99.

732

733

von Mering C, Zdobnov EM, Tsoka S, Ciccarelli FD, Pereira-Leal JB, Ouzounis CA, and Bork

734

P. 2003. Genome evolution reveals biochemical networks and functional modules. Proc Natl

735

Acad Sci 100(26):15428-15433.

736

737

Wang X, Castro MA, Mulder KW, and Markowetz F. 2012. Posterior Association Networks and

738

Functional Modules Inferred from Rich Phenotypes of Gene Perturbations. PLoS Comput Biol

739

8(6):e1002566.

740

741

Wang YC and Chen BS. 2010. Integrated cellular network of transcription regulations and

protein-protein interactions. BMC Systems Biology 4:20.

743

744

Watanabe K, Stringer S, Frei O, Mirkov MU, de Leeuw C, Polderman TJC, van der Sluis S,

Andreassen OA, Neale BM, and Posthuma D, et al.. 2019. A global overview of pleiotropy and

genetic architecture in complex traits. Nat Genet 51(9):1339-1348.

747

748 Wernicke S and Rasche F. 2006. FANMOD: a tool for fast network motif detection.

749

Bioinformatics 22(9):1152-1153.

750

Peer] reviewing PDF | (2021:11:67599:2:0:NEW 28 Jan 2022) 
751 Wolf YI, Katsnelson MI, and Koonin EV. 2018. Physical foundations of biological complexity.

752 Proc Acad Natl Sci 115(37):E8678-E8687.

753

754 Wolfger H, Mamnun YM, and Kuchler K. 2004. The yeast Pdr15p ATP-binding cassette (ABC)

755 protein is a general stress response factor implicated in cellular detoxification. J Biol Chem

756 279(12):11593-9.

757

758 Wray NR, Wijmenga C, Sullivan PF, Yang J, and Visscher PM. 2018. Common Disease Is More 759 Complex Than Implied by the Core Gene Omnigenic Model. Cell 173(7):1573-1580.

760

761 Yu H, Braun P, Yildririm M, Lemmens I, Venkatesan K, Sahalie J, Hirozane-Kishikawa T, 762 Gebreab F, Li N, Simonis N, et al.. 2008. High-Quality Binary Protein Interaction Map of the 763 Yeast Interactome Network. Science 322(5898):104-110.

764

765 Zanudo Tejeda JG, Yang G, and Albert R. 2017. Structure-based control of complex networks 766 with nonlinear dynamics. Proc Acad Natl Sci 114(28):7234-7239. 


\section{Figure 1}

Definition of functional network motifs (FNMs).

(A) Exemplary schematic of an FNM. A Protein-protein interaction (PPI) between proteins $A$ and $B$ occurs through direct physical contact of the two proteins and is often functional. $A$ genetic interaction $(\mathrm{Gl})$ between the coding genes $a$ and $b$ is derived from assessing the fitness, normally estimated through a growth phenotype and here schematically illustrated by a circle representing the cell colony size, of $a b \Delta$ double-deletion strains relative to expectation based on the effect of the single deletions $a \Delta$ and $b \Delta$ on cell fitness. Significantly less severe and more severe deviations from expected are recorded as positive and negative Gls. Here, FNMs of pre-determined sizes are generated by integrating the genome-wide PPI and GI networks of S.cerevisiae. Black edges represent PPIs and define a graphlet. Green edges represent genetic Gls. The source node as the starting node of the motif search by depth-first-search and most distant layer in the motif are highlighted. A network motif becomes an FNM if Gls are present in at least $50 \%$ of all non-self edges, and between the source node and all nodes in the most distant layer. (B) Counts of conventional PPI network motifs and FNMs as function of the motif size k. Gls introduce selectivity and the occurrences of FNMs are about two orders of magnitude lower than that of classical network motifs. (C) Counts of motifs in the yeast protein and genetic networks for the most represented motif topologies. Shown are the total motif counts based only on the PPI network (PPI), for randomized PPI networks (PPI random), as well as for FNMs and and FNMs computed from randomized GI network (FNM random). Motif topologies are indicated both graphically and in compressed graph6 format. 
A

C
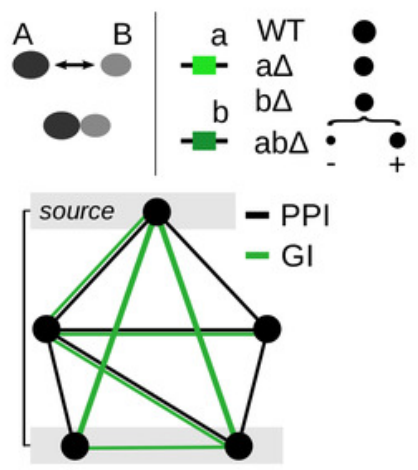

B
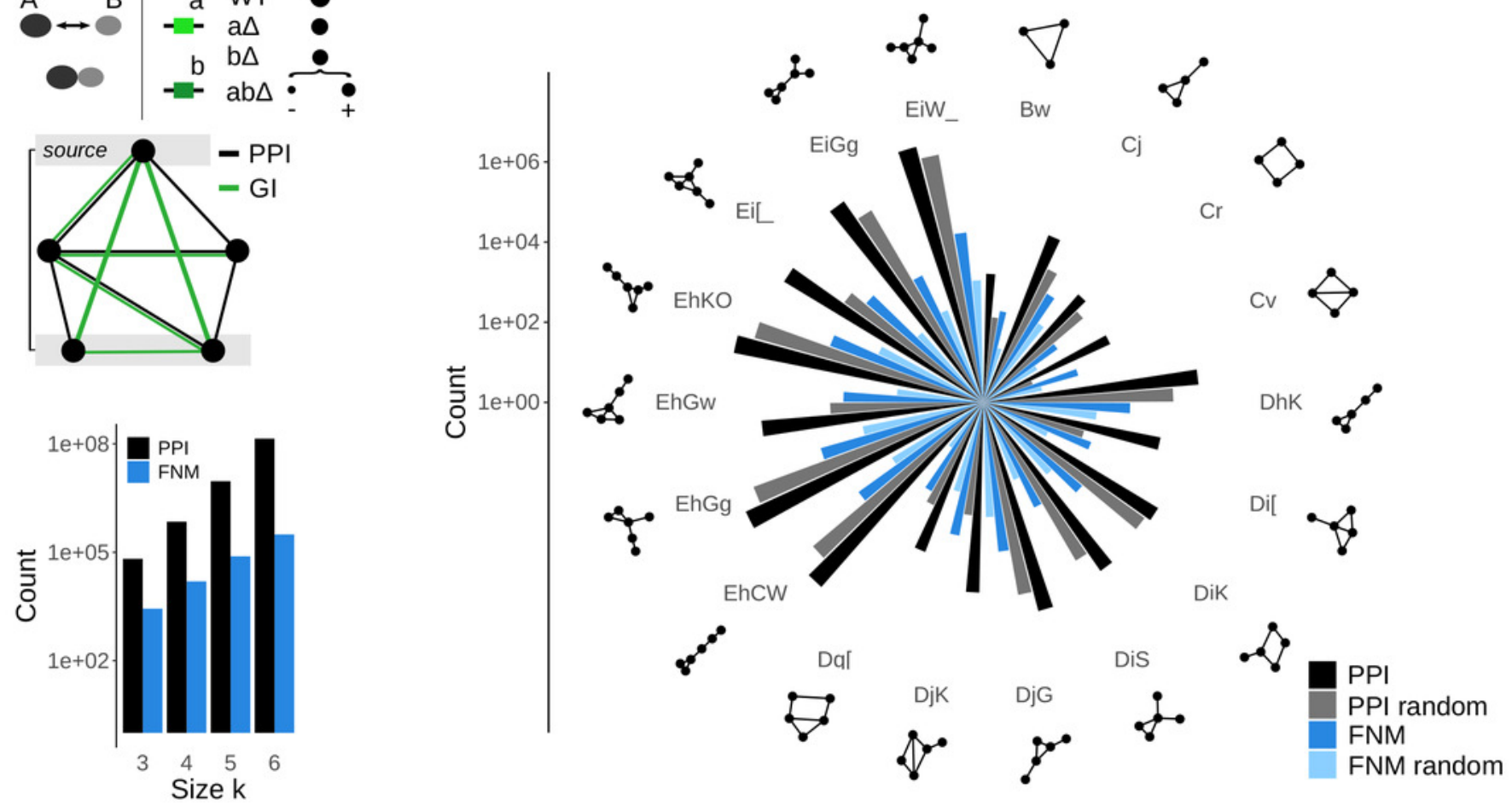


\section{Figure 2}

FNMs are enriched in functionally important and related genes.

(A) Fractions of FNMs and classical network motifs (PPI) that contain suppressor interactions as proxy for functionally related genes. As a control (CTRL) are shown the rate of suppresor interactions in randomized motifs with the same content of Gls as FNMs. (B) Fractions of essential genes and genes coding for protein complex subunits in FNMs and in classical network motifs (PPI). (C) Numbers of FNMs that do not cluster compared to all FNM, together with the number of genes (ORFs) in all FNMs and only in those that do not cluster. (D) Distributions of the number of motifs per gene for the genes in FNMs that cluster vs. those that do not cluster together with the network degree for the same groups of genes. 
A
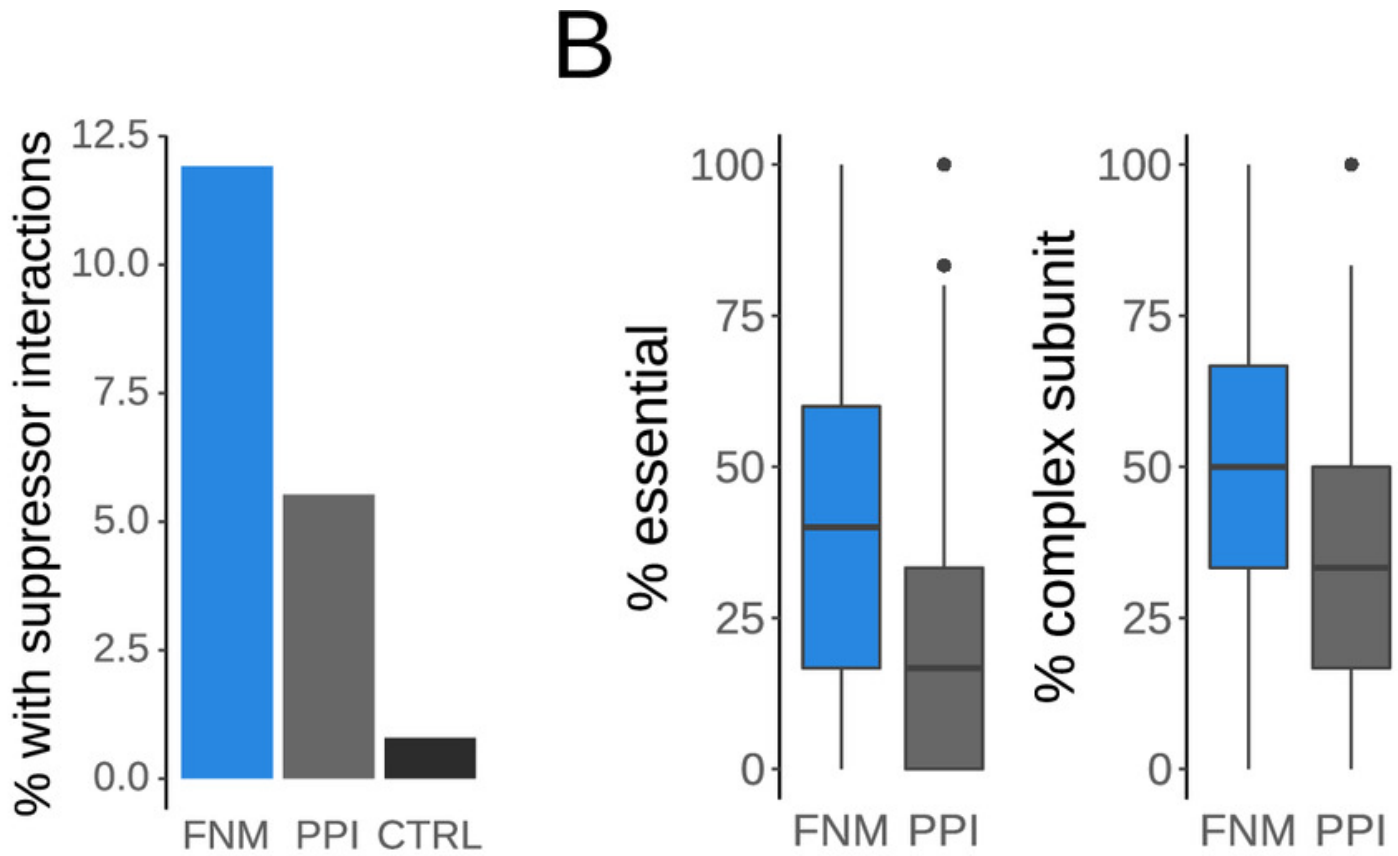

FNM PPI

FNM PPI

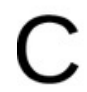

D
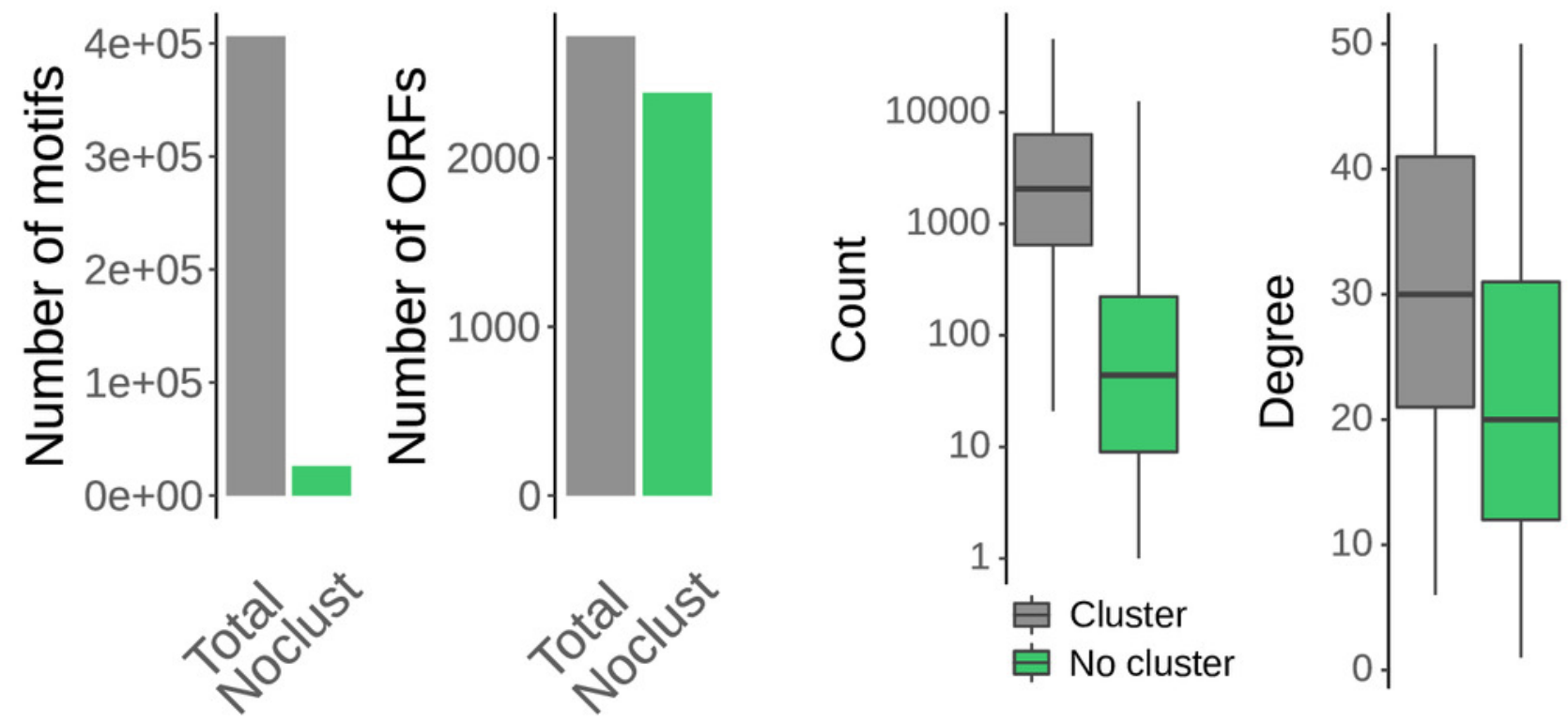


\section{Figure 3}

Genetic interactions in functional network motifs.

(A) Fractions of positive (yellow) and negative (blue) genetic interactions in FNMs. (B)

Consensus genetic interactions between protein complexes. (C) Consensus PPI and GI map of yeast protein complexes. PPIs are shown with straight black lines, positive Gls in yellow and negative Gls in blue. The line width reflects the number of interactions observed between the complex pair. (D) Fractions of pairwise interactions between protein complexes that included both PPIs and GIs, only PPIs and only Gls. (E) Exemplary FNM that connects six different protein complexes involved in DNA replication and repair. Both the gene and complex names are indicated.

A
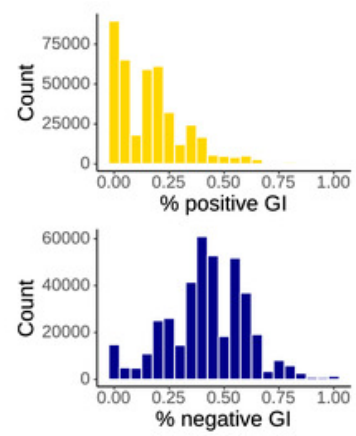

B

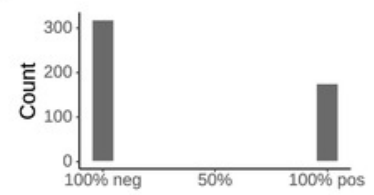

C

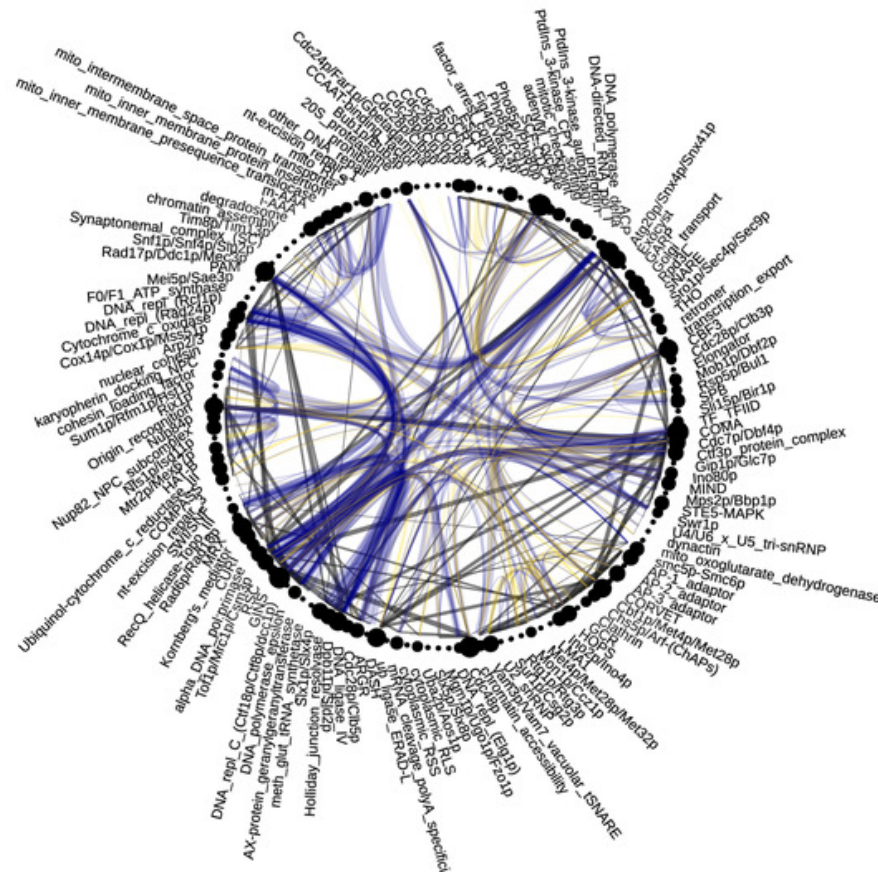

D

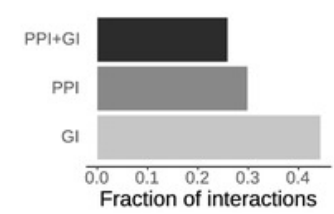

E

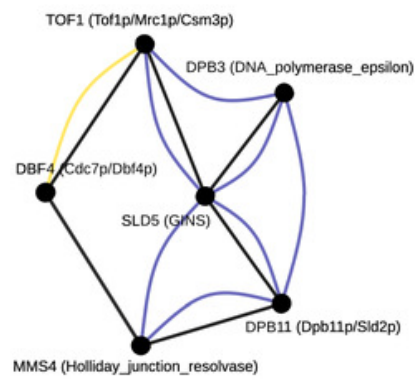




\section{Figure 4}

FNMs identify known, and novel candidates for feedback and cross-talk interactions.

(A) Yeast protein complex interaction network based on the interactions in the FNMs. Protein complex subunits are merged into joint nodes (black squares). Auxiliary, non-complex, nodes (purple circles) are scaled based on their betweenness centrality. (B) Distributions of the betweenness centrality of the auxiliary nodes connecting protein complexes. Shown are the values for the network derived from the FNMs, from the PPI network with $\mathrm{d}$ max $<50$ as used to derive the FNMs, and from the full PPI network (PPI all). (C) Network sizes of the FNM, PPI and PPI all networks. Also shown are the effective network sizes that only include auxiliary nodes with non-zero betweenness centralities (dashed borders). (D) Ranking of the auxiliary nodes with the highest betweenness centrality in the FNM network. (E) All FNMs that connect the yeast consensus transcription factors to the yeast metabolic network. 


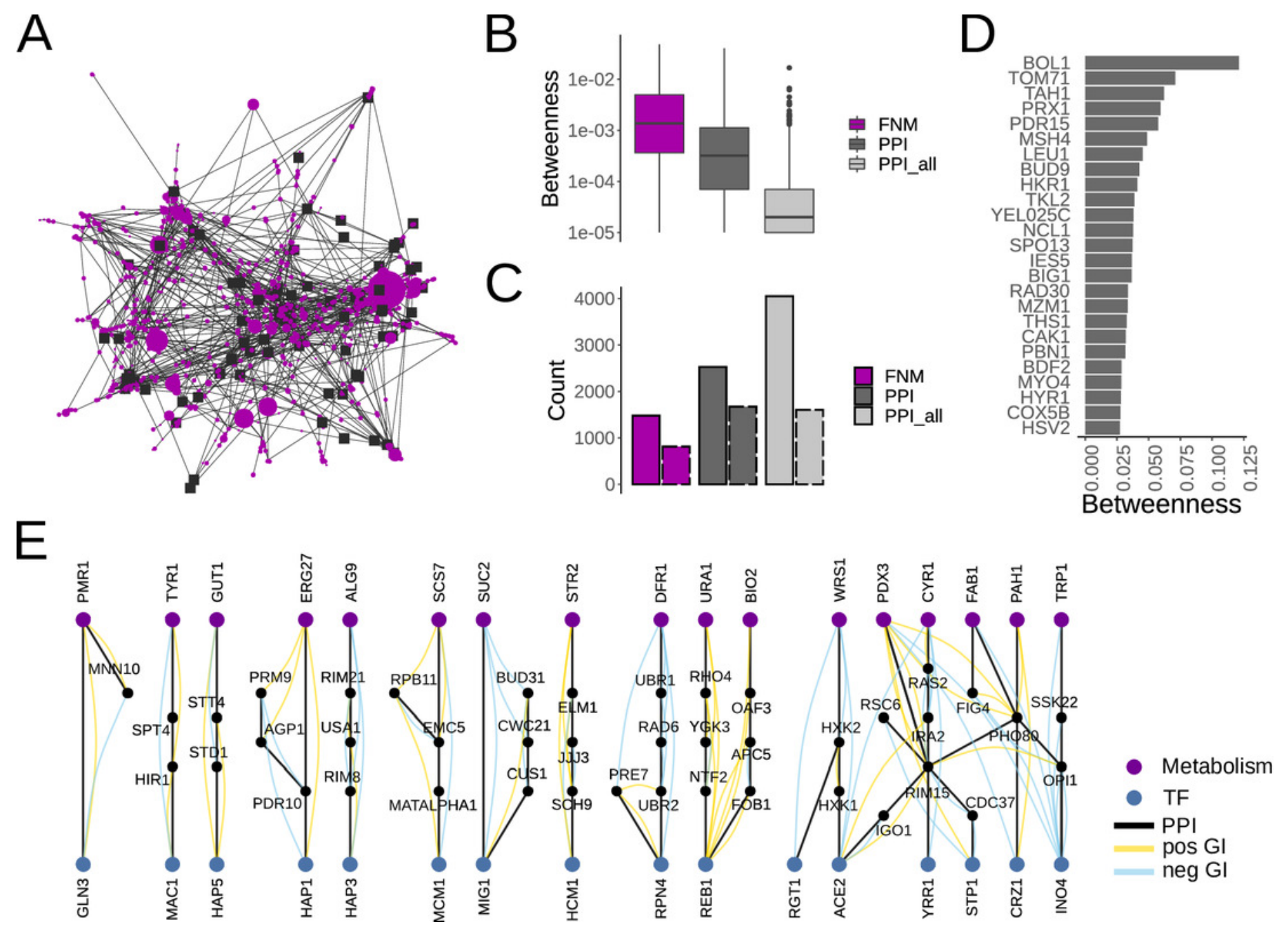




\section{Figure 5}

Transcriptional co-regulation of FNMs in response to environmental perturbations.

(A) Heatmap of co-regulation expressed through average pairwise cosine similarity of the expression profiles of the individual nodes in the motif. The top $5 \%$ and $1 \%$ are color coded. Rows of motifs with no co-regulation in at least the top $5 \%$ are not shown. (B) Distribution of the FNM co-regulation scores. The top $5 \%$ and $1 \%$ are indicated in the far right tail of the distribution. (C) Cumulative distribution curves of the co-regulation scores as function of motif size k. (D) An exemplary cluster of motifs that is strongly transcriptionally co-regulated during heat stress identifies the core splicing machinery. (E) An exemplary FNM that is strongly co-regulated during heat stress highlights extensive cross-talk between RNA surveillance and quality control. (F) An FNM that connects the transcription regulatory network to the metabolic network may indicate a regulatory function of the E2-E3 complexes formed by RAD6, UBR1 and UBR2 in coupling transcript and protein levels during heat stress.

A

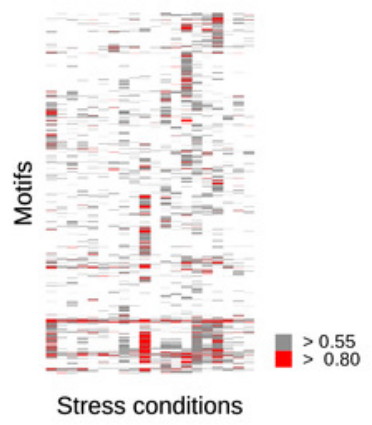

B

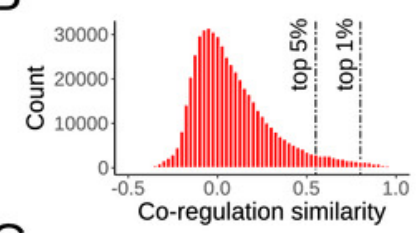

$\mathrm{C}$

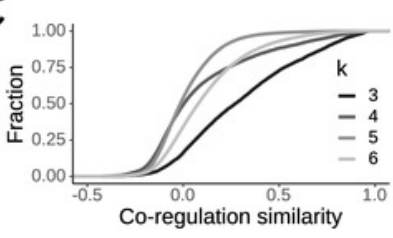

$\mathrm{D}$

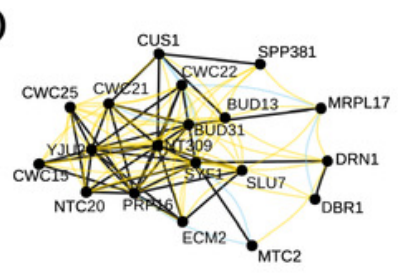

$E$

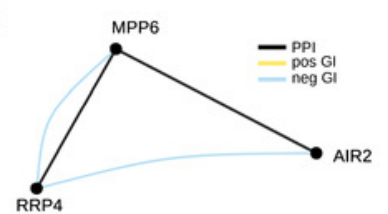

$\mathrm{F}$
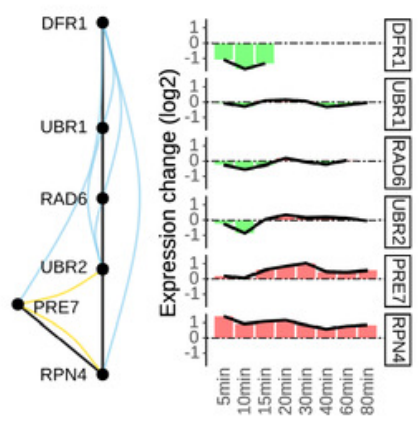